\title{
ON THE CONVERGENCE OF MULTIPLE FOURIER SERIES
}

\author{
BY CHARLES FEFFERMAN
}

Communicated by M. H. Protter, January 11, 1971

We continue from [2].

THEOREM. Let $P$ be an open polygonal region in $R^{2}$, containing the origin. Set $\lambda P=\{(\lambda x, \lambda y) \mid(x, y) \in P\}$ for $\lambda>0$. Then for

$$
f \sim \sum_{m, n=-\infty}^{\infty} a_{m n} \exp [i(m x+n y)]
$$

in $L^{2}([0,2 \pi] \times[0,2 \pi])$, we have

$$
f(x, y)=\lim _{\lambda \rightarrow \infty} \quad \sum_{(m, n) \in \lambda P} a_{m n} \exp [i(m x+n y)]
$$

almost everywhere.

Surprisingly, this is an easy consequence of Carleson's theorem [1] on convergence of Fourier series of one variable.

Proof. It is enough to prove the maximal inequality

$$
\left\|\sup _{\lambda}\left|\sum_{(m, n) \in \lambda P} a_{m n} \exp [i(m x+n y)]\right|\right\|_{2} \leqq C\|f\|_{2} .
$$

Inequality (1) follows from the special case in which $P$ is a triangle with a vertex at the origin; for any polygon breaks up into triangles, and the characteristic function of any triangle is a linear combination of characteristic functions of triangles with vertices at zero. Consequently, we can assume $P$ has the form $P=\{(x, y) \in S \mid(x, y) \cdot t<a\}$, where $S$ is a sector of angle $<\pi$ emanating from the origin, $t \in R^{2}$, and $a \in R^{1}$. Thus (1) is equivalent to

$$
\left\|\sup _{b \in R^{1}}\left|\sum_{(m, n) \in S ;(m, n) \cdot t<b} a_{m n} \exp [i(m x+n y)]\right|\right\|_{2} \leqq C\|f\|_{2} .
$$

Evidently, it suffices to prove (2) for rational $t$ (with $C$ independent of $t$ ), and to do so it is clearly enough to deal with the case $t=(p, q)$ where $p$ and $q$ are relatively prime integers. Finding integers $r$ and $s$ for which $p r-q s=1$, we let the matrix $A=\left(\begin{array}{c}p \\ a \\ c\end{array}\right) \in S L(2, Z)$ act as an automorphism of the 2-torus. Under the action of $A$, (2) becomes

AMS 1969 Subject classifications. Primary 4211, 4240. 


$$
|| \sup _{b}\left|\sum_{\left(m^{\prime}, n^{\prime}\right) \in S^{\prime} ; m^{\prime}<b} a_{m^{\prime} n^{\prime}} \exp \left[i\left(m^{\prime} x^{\prime}+n^{\prime} y^{\prime}\right)\right]\right|\left\|_{2} \leqq C\right\| f^{\prime} \|_{2} .
$$

Here,

$$
S^{\prime}=A^{-1}(S), f^{\prime}\left(x^{\prime}, y^{\prime}\right)=f\left(A\left(x^{\prime}, y^{\prime}\right)\right) \text { and } \sum_{m^{\prime}, n^{\prime}} a_{m^{\prime} n^{\prime}} \exp \left[i\left(m^{\prime} x^{\prime}+n^{\prime} y^{\prime}\right)\right]
$$

is the Fourier series of $f^{\prime}$. Note that $C$ is unchanged from (2) to (3). However, (3) follows at once by applying the Carleson-Hunt theorem of [3] to the function $g\left(\cdot, y^{\prime}\right)$ for each $y^{\prime}$, where $g^{\prime}\left(x^{\prime}, y^{\prime}\right)$ $\sim \sum_{\left(m^{\prime}, n^{\prime}\right) \in S^{\prime}} a_{m^{\prime} n^{\prime}} \exp \left[i\left(m^{\prime} x^{\prime}+n^{\prime} y^{\prime}\right)\right]$. Q.E.D.

REMARKS. 1. The same proof applies to all $L^{p}, p>1$, and also (with some padding) to polyhedra in $n$ variables.

2. For $P$ a rectangle, a more precise argument, discovered independently by P. Sjölin [4], proves convergence of double Fourier series under minimal growth conditions on $f$. The best known hypotheses are $f \in L(\log L)^{2} \log \log L$ for $P$ a rectangle, and $f \in L(\log L)^{3} \log \log L$ in general. The relationship of our proof to Sjölin's is not clear.

3. N. Tevzadze [5] has shown that for $f \in L^{2}([0,2 \pi] \times[0,2 \pi])$ and for any monotone sequence of rectangles $R_{1} \subseteq R_{2} \subseteq R_{8} \subseteq \ldots$ in $R^{2}$ with sides parallel to the coordinate axes,

$$
f(x, y)=\lim _{i \rightarrow \infty} \sum_{(m, n) \in R_{i}} a_{m n} \exp [i(m x+n y)]
$$

almost everywhere.

Compare with the counterexamples of [2].

\section{REFERENCES}

1. L. Carleson, On convergence and growth of partial sums of Fourier series, Acta Math. 116 (1966), 135-157. MR 33 \#7774.

2. C. Fefferman, On the divergence of multiple Fourier series, Bull. Amer. Math. Soc. 77 (1971), 191-195.

3. R. A. Hunt, On the convergence of Fourier series, Proc. Conf. on Orthogonal Expansions and their Continuous Analogues (Edwardsville, Ill., 1967), Southern Illinois Univ. Press, Carbondale, Ill., 1968, pp. 235-255. MR 38 \#6296.

4. P. Sjölin, On the convergence almost everywhere of certain singular integrals and multiple Fourier series, Ark. Mat. 9 (1971) (to appear).

5. N. Tevzadze, On the convergence of double Fourier series of quadratic summable functions, Soobšc. Akad. Nauk Gruzin. SSR 1970, 277-279.

University of Chicago, Chicago, Illinois 60637 